\title{
Stability Solution of the Nonlinear Schrödinger Equation
}

\author{
Mujahid Abd Elmjed M-Ali \\ Department of Mathematics, Faculty of Education, Kassala University, Kassala, Sudan \\ Email: mujahid@mail.ustc.edu.cn
}

Received February 19, 2013; revised March 28, 2013; accepted April 30, 2013

Copyright (C) 2013 Mujahid Abd Elmjed M-Ali. This is an open access article distributed under the Creative Commons Attribution License, which permits unrestricted use, distribution, and reproduction in any medium, provided the original work is properly cited.

\begin{abstract}
In this paper we discuss stability theory of the mass critical, mass-supercritical and energy-subcritical of solution to the nonlinear Schrödinger equation. In general, we take care in developing a stability theory for nonlinear Schrödinger equation. By stability, we discuss the property: the approximate solution to nonlinear Schrödinger equation $\tilde{u}$ obeying $\left(i \partial_{t}+\Delta\right) \tilde{u}=F(\tilde{u})+e$ with $e$ small in a suitable space and $\tilde{u}_{0}-u_{0}$ small in $\dot{H}_{x}^{s_{c}}$ and then there exists a veritable solution $u$ to nonlinear Schrödinger equation which remains very close to $\tilde{u}$ in critical norms.
\end{abstract}

Keywords: NLS; Wellposed

\section{Introduction}

In this paper, we study the stability theory of solutions to the nonlinear Schrödinger equation (NLS).

We consider the Cauchy problem for the nonlinear Schrödinger equation

$$
\left\{\begin{array}{l}
i u_{t}+\Delta u=\mu F(u) \\
u(0, x)=u_{0}(x)
\end{array}\right\}
$$

where $F(u)=|u|^{p} u, \quad \mu= \pm 1$ the solution $u=u(t, x)$ is a complex-valued function in $\mathbb{R}^{d} \times \mathbb{R}$.

The Equation (1.1) is called mass-critical or $L^{2}$ critical if $p=\frac{4}{d}$, and it is called mass-supercritical and energy-subcritical when $p<\frac{4}{d-2}$.

The solutions to (1.1) have the invariant scaling

$$
u(x, t) \mapsto \lambda^{\frac{2}{p}} u\left(\lambda x, \lambda^{2} t\right)
$$

Definition 1.1 (Solution) Let $I \subseteq \mathbb{R}$ such that $0 \in I$. A function $u: I \times \mathbb{R}^{d} \rightarrow \mathbb{C}$ is a strong solution to (1.1) if and only if it belongs to $C\left(I, H^{1}\left(\mathbb{R}^{d}\right)\right)$, and for all $t \in I$ satisfies the integral equation

$$
u(t)=\mathrm{e}^{i t \Delta} u_{0}+i \mu \int_{0}^{t} \mathrm{e}^{i(t-r) \Delta} F(u(r)) \mathrm{d} r .
$$

A function $u: I \times \mathbb{R}^{d} \rightarrow \mathbb{C}$ is a weak solution to (1.1) if and only if $u \in L^{\infty}\left(I, H^{1}\left(\mathbb{R}^{d}\right)\right)$, and for all $t \in I$ satisfies the integral Equation (1.3).

The solutions to (1.1) have the mass

where

$$
M[u](t)=M\left[u_{0}\right]
$$

$$
M[u](t)=\int_{\mathbb{R}^{d}}|u(x, t)|^{2} \mathrm{~d} x .
$$

Energy $E[u](t)=E\left[u_{0}\right]$ where,

$$
E[u](t)=\frac{1}{2} \int_{\mathbb{R}^{d}}|\nabla u(x, t)|^{2} \mathrm{~d} x-\frac{\mu}{p+2} \int_{\mathbb{R}^{d}}|u(x, t)|^{p+2} \mathrm{~d} x .
$$

Definition 1.2 The problem (1.1) is locally wellposed in $H^{1}\left(\mathbb{R}^{d}\right)$ if for any $u \in H^{1}\left(\mathbb{R}^{d}\right)$ there exist a time $T>0$ and an open ball $B$ in $H^{1}\left(\mathbb{R}^{d}\right)$ such that $u_{0} \in B$, and a subset $X$ of $C\left([-T, T], H^{1}\left(\mathbb{R}^{d}\right)\right)$, such that for each $u_{0} \in B$ there exists a unique solution $u \in X$ to the Equation (1.3), and furthermore, the map $u_{0} \rightarrow u$ is continuous from $B$ to $X$. If $T$ can be taken arbitrarily large $(T=+\infty)$, the problem is globally wellposed.

Definition 1.3 A global solution $u(t)$ to (1.1) is scattering in $H^{s}\left(\mathbb{R}^{d}\right)$ as $t \rightarrow+\infty$ if there exists $\psi^{+} \in H^{s}\left(\mathbb{R}^{d}\right)$ such that

$$
\lim _{t \rightarrow+\infty}\left\|u(t)-\mathrm{e}^{i t \Delta} \psi^{+}\right\|_{H^{s}\left(\mathbb{R}^{d}\right)}=0 .
$$

Similarly, we can define scattering in $H^{s}\left(\mathbb{R}^{d}\right)$ for 
$t \rightarrow-\infty$.

For more definition of critical case see [1-3].

In this paper we discuss stability theory of the mass critical, mass-supercritical and energy-subcritical of solution to the nonlinear Schrödinger equation. In section three we discuss the stability of the mass critical solutions and in section four mass-supercritical and energysub-critical solutions are discussed.

Theorem 1.1 Let $u_{0} \in L_{x}^{2}\left(\mathbb{R}^{d}\right)$ and $t_{0} \in \mathbb{R}$. Then there exists a unique maximal-lifespan solution $u: I$ $\times \mathbb{R}^{d} \rightarrow C$ to (1.1) with $t_{0} \in I$ and initial data $u\left(t_{0}\right)=u_{0}$. Moreover:

1) The interval $I$ is an open subset of $\mathbb{R}$.

2) For all $t \in I$, we have $M(u(t))=M\left(u_{0}\right)$ so, we define $M(u)=M(u(t))$.

3) If the solution $u$ does not blow up forward in time, then $\sup I=+\infty$, and moreover $u$ scatters forward in time to $\mathrm{e}^{i t \Delta} u_{+}$for some $u_{+} \in L_{x}^{2}\left(\mathbb{R}^{d}\right)$. Conversely, if $u_{+} \in L_{x}^{2}\left(\mathbb{R}^{d}\right)$ then there exists a unique maximallifespan solution $u$ which scatters forward in time to $\mathrm{e}^{i t \Delta} u_{+}$.

4) If the solution $u$ does not blow up backward in time, then inf $I=-\infty$ and moreover $u$ scatters backward in time to $\mathrm{e}^{i t \Delta} u_{-}$for some $u_{-} \in L_{x}^{2}\left(\mathbb{R}^{d}\right)$. Conversely, if $u_{-} \in L_{x}^{2}\left(\mathbb{R}^{d}\right)$ then there exists a unique maximallifespan solution $u$ which scatters backward in time to $\mathrm{e}^{i t \Delta} u$

5) If $M(u) \leq c_{d}^{-1}$ where a constant $c_{d}>0$ depending only on $d$ then

$$
S(u) \leq c_{d} M(u)^{(d+2) / d} .
$$

In particular, no blowup occurs and we have global existence and scattering both ways.

6) For every $A>0$ and $\varepsilon>0$ there exists $\delta>0$. With property: if $u: I \times \mathbb{R}^{d} \rightarrow C$ is a solution (not necessarily maximal-lifespan) such that

$M(u), S(u) \leq A$ and $t_{0} \in I, \quad v_{0} \in L_{x}^{2}\left(\mathbb{R}^{d}\right)$ are such that $M\left(u\left(t_{0}\right)-v_{0}\right) \leq \delta$, then there exists a solution $v: I \times \mathbb{R}^{d} \rightarrow C$ with $v\left(t_{0}\right)=v_{0}$ such that $S(u-v) \leq \varepsilon$ and $M(u(t)-v(t)) \leq \varepsilon$ for all $t \in I$.

For proof: See [4-6].

Now in the following we will discuss Standard local well-posedness theorem.

Theorem 1.2 Let $d \geq 1, s_{c} \geq 0$ and let $u_{0} \in H_{x}^{s_{c}}\left(\mathbb{R}^{d}\right)$. Assume that $s_{c}<1+p$ if $p$ is not an even integer. Then there exists $\eta_{0}=\eta_{0}(d)$ such that if between $0<\eta \leq \eta_{0}$ and $I$ there is a compact interval containing zero such that

$$
\left\||\nabla|^{s_{c}} \mathrm{e}^{i t \Delta} u_{0}\right\|_{L_{t}^{p+2} L_{x}^{2(d-2)+d p}\left(I \times \mathbb{R}^{d}\right)} \leq \eta
$$

then there exists a unique solution $u$ to $(1.1)$ on $I \times \mathbb{R}^{d}$. Furthermore, we have the bounds

$$
\begin{aligned}
& \left\||\nabla|^{s_{c}} u\right\|_{L_{t}^{p+2} \frac{2 d(p+2)}{L_{x}^{2(d-2)+d p}}\left(I \times \mathbb{R}^{d}\right)} \leq 2 \eta \\
& \left\||\nabla|^{s_{c}} u\right\|_{S^{0}\left(I \times \mathbb{R}^{d}\right)} \lesssim\left\|\left.\nabla\right|^{s_{c}} u_{0}\right\|_{L_{x}^{2}}+\eta^{1+p} \\
& \|u\|_{S^{0}\left(I \times \mathbb{R}^{d}\right)} \lesssim\left\|u_{0}\right\|_{L_{x}^{2}}
\end{aligned}
$$

where $S^{0}\left(I \times \mathbb{R}^{d}\right)$ for the closure of all test functions under this norm.

\section{Strichartz Estimate}

In this section we discus some notation and Strichartz estimate.

\subsection{Some Notation}

We write $X \lesssim Y$ anywhere in this work whenever there exists a constant $c$ independent of the parameters, so that $X \leq c Y$. The shortcut $O(X)$ denotes a finite linear gathering of terms that "look like" $X$, but possibly with some factors changed by their complex conjugates.

We start by the definition of space-time norms

$$
\|u\|_{L_{t}^{q} L_{x}^{r}\left(R \times R^{3}\right)}=\|u\|_{L_{t}^{q} L_{x}^{r}}:=\left(\int_{\mathbb{R}}\left(\int_{\mathbb{R}^{d}}|u(x, t)|^{r} \mathrm{~d} x\right)^{\frac{q}{r}} \mathrm{~d} t\right)^{\frac{1}{q}}
$$

The inhomogeneous Sobolev norm $H^{s}\left(\mathbb{R}^{d}\right)$ (when $s$ is an integer) is defined by:

$$
\|f\|_{H^{s}\left(\mathbb{R}^{d}\right)}=\|f\|_{H^{s}}:=\sum_{|\alpha|=0}^{s}\left\|\partial_{x}^{\alpha} f\right\|_{L^{2}\left(\mathbb{R}^{d}\right)}
$$

When $s$ is any real number as

$$
\|f\|_{H^{s}\left(\mathbb{R}^{d}\right)}=\|f\|_{H^{s}}:=\left(\int_{\mathbb{R}^{d}}|\hat{f}(\xi)|^{2}\left(1+|\xi|^{2}\right)^{s} \mathrm{~d} \xi\right)^{\frac{1}{2}}
$$

The homogeneous Sobolev norm $\dot{H}^{s}\left(\mathbb{R}^{d}\right)$ defines as:

$$
\|f\|_{\dot{H}^{s}\left(\mathbb{R}^{d}\right)}=\|f\|_{\dot{H}^{s}}:=\left(\int_{\mathbb{R}^{d}}|\hat{f}(\xi)|^{2}|\xi|^{2 s} \mathrm{~d} \xi\right)^{\frac{1}{2}}
$$

For any space time slab $K \times R^{d}$,

We use $L_{t}^{q} L_{x}^{r}\left(K \times R^{d}\right)$ to denote the Banach space of function $K \times R^{d} \rightarrow C$ whose norm is

$$
\|u\|_{L_{t}^{q} L_{x}^{r}\left(K \times R^{d}\right)}:=\left(\int_{K} u(t)_{L_{x}^{r}}^{q} \mathrm{~d} t\right)^{\frac{1}{q}}<\infty .
$$

With the usual adjustments when $q$ or $r$ is equal to infinity. When $q=r$ we abbreviate $L_{t}^{q} L_{x}^{r}$ as $L_{t, x}^{q}$.

A Gagliardo-Nirenberg type inequality for Schrödinger equation the generator of the pseudo conformal 
transformation $J=x-2 i t \partial$ plays the role of partial differentiation.

\subsection{Strichartz Estimate}

Definition 2.1 The exponent pair $(q, r)$ is says the Schrödinger-admissible if

$$
\begin{aligned}
& d \geq 1, \text { and } 2 \leq q, r \leq \infty \\
& \frac{1}{q}=\frac{d}{2}\left(\frac{1}{2}-\frac{1}{r}\right), \quad(q, r, d) \neq(2, \infty, 2) .
\end{aligned}
$$

Definition 2.2 The exponent pair $(q, r)$ is says the Schrödinger-acceptable if

$$
\begin{aligned}
& 1 \leq q<\infty, 2 \leq r \leq \infty, \frac{1}{q}<d\left(\frac{1}{2}-\frac{1}{r}\right) \\
& \text { or }(q, r)=(\infty, 2) .
\end{aligned}
$$

Let $\mathrm{e}^{i t \Delta}$ be the free Schrödinger evolution. From the explicit formula

$$
\mathrm{e}^{i t \Delta} f(x)=\frac{1}{(4 \pi i t)^{\frac{d}{2}}} \int_{\mathbb{R}^{d}} \mathrm{e}^{\frac{i|x-y|^{2}}{4 t}} f(y) \mathrm{d} y,
$$

we obtain the standard dispersive inequality

$$
\left\|\mathrm{e}^{i t \Delta} f(x)\right\|_{L_{x}^{\infty}\left(\mathbb{R}^{d}\right)} \precsim|t|^{-\frac{d}{2}}\|f\|_{L_{x}^{1}\left(\mathbb{R}^{d}\right)},
$$

for all $t \neq 0$.

In particular, as the free propagator conserves the $L_{x}^{2}$ norm,

$$
\left\|\mathrm{e}^{i t \Delta} f(x)\right\|_{L_{x}^{p}\left(\mathbb{R}^{d}\right)} \precsim|t|^{d\left(\frac{1}{P}-\frac{1}{2}\right)}\|f\|_{L_{x}^{p^{\prime}}\left(\mathbb{R}^{d}\right)}
$$

For all $t \neq 0$ and $2 \leq p \leq \infty$,

$$
\text { where } \frac{1}{p}+\frac{1}{p^{\prime}}=1 \text {. }
$$

If $u: I \times \mathbb{R}^{d} \rightarrow C$ solves the inhomogeneous Equation (1.1) for some

$$
t_{0} \in I, u_{0} \in L_{x}^{2}\left(\mathbb{R}^{d}\right) \text { and } F \in L_{t, x}^{2(d+2) /(d+4)}\left(I \times \mathbb{R}^{d}\right)
$$

in the integral.

Duhamel (1.3). Then we have

$$
\begin{aligned}
& \|u\|_{C_{t}^{0} L_{x}^{2}\left(I \times \mathbb{R}^{d}\right)}+\|u\|_{L_{t, x}^{2(d+2) / d}\left(I \times \mathbb{R}^{d}\right)} \\
& \leq C_{d}^{\prime \prime}\left(\left\|u_{0}\right\|_{L_{x}^{2}\left(\mathbb{R}^{d}\right)}+\|F\|_{L_{t, x}^{2(d+2) /(d+4)}\left(I \times \mathbb{R}^{d}\right)}\right),
\end{aligned}
$$

for some constant $0<C_{d}^{\prime \prime}<\infty$ depending only on the dimension $d$.

For some constant $C_{d}^{\prime}$ depending only on $d$ we have the Holder inequality

$$
\begin{aligned}
& \|F(u)-F(v)\|_{L_{t, x}^{2(d+2) /(d+4)}\left(I \times \mathbb{R}^{d}\right)} \leq C_{d}^{\prime}\|u-v\|_{L_{t, x}^{2(d+2) / d}\left(I \times \mathbb{R}^{d}\right)} \\
& \times\left[\|u\|_{L_{t, x}^{2(d+2) / d}\left(I \times \mathbb{R}^{d}\right)}+\|v\|_{L_{t, x}^{2(d+2) / d}\left(I \times \mathbb{R}^{d}\right)}\right]^{\frac{4}{d}} .
\end{aligned}
$$

We now return to prove Theorem 1.2.

Proof Theorem 1.2 The theorem follows from a contraction mapping argument. More accurate, defined

$$
\Phi(u)(t):=\mathrm{e}^{i t \Delta} u_{0}-i \int_{0}^{t} \mathrm{e}^{i(t-s) \Delta} F(u(s)) \mathrm{d} s,
$$

using the Strichartz estimates, we will show that the map $u \rightarrow \Phi(u)$ is a contraction on the set $B_{1} \cap B_{2}$ where

$$
\begin{aligned}
& B_{1}:=\left\{u \in L_{t}^{\infty} H_{x}^{s_{c}}\left(I \times \mathbb{R}^{d}\right):\|u\|_{L_{t}^{\infty} H_{x}^{s_{c}}\left(I \times \mathbb{R}^{d}\right)}\right. \\
& \left.\leq 2\left\|u_{0}\right\|_{H_{x}^{s_{c}}}+C(d, p)(2 \eta)^{1+p}\right\} \\
& B_{2}:=\left\{u \in L_{t}^{p+2} W_{x}^{s_{c}, \frac{2 d(p+2)}{2(d-2)+d p}}\left(I \times \mathbb{R}^{d}\right)\right. \\
& :\left\||\nabla|^{s_{c}} u\right\|_{L_{t}^{p+2} L_{x}^{2(d-2)+d p}\left(I \times \mathbb{R}^{d}\right)}^{\frac{2 d(p+2)}{2(d-1)}} \\
& \left.\leq 2 \eta \text { and }\|u\|_{L_{t}^{p+2} L_{x}^{2 d(d-2)+d p}\left(I \times \mathbb{R}^{d}\right)} \leq 2 C(d, p)\left\|u_{0}\right\|_{L_{x}^{2}}\right\}
\end{aligned}
$$

under the metric given by

$$
d(u, v):=\|u-v\|_{L_{t}^{p+2}} \frac{2 d(p+2)}{L_{x}^{2(d-2)+d p}}\left(I \times \mathbb{R}^{d}\right) .
$$

Here $C(d, p)$ denotes a constant that changes from line to line. Note that the norm appearing in the metric scales like $L_{x}^{2}$. Note also that both $B_{1}$ and $B_{2}$ are closed (and hence complete) in this metric.

Using the Strichartz inequality and Sobolev embedding, we find that for $u \in B_{1} \cap B_{2}$

$$
\begin{aligned}
& \|\Phi(u)\|_{L_{t}^{\infty} H_{x}^{s_{c}}\left(I \times \mathbb{R}^{d}\right)} \\
& \left.\leq\left\|u_{0}\right\|_{H_{x}^{s_{c}}}+C(d, p)\left\|\nabla^{s_{c}} F(u)\right\|_{L_{t}^{p+1} \frac{p+2}{L_{x}^{2(d+2)+d p}}\left(I \times \mathbb{R}^{d}\right)}^{\frac{2 d(p+2)}{2(d+2)}}\right) \\
& \leq\left\|u_{0}\right\|_{H_{x}^{s_{c}}}+\left(C(d, p)\left\|\langle\nabla\rangle^{s_{c}} u\right\|_{L_{t}^{p+2} \frac{2 d(p+2)}{L_{x}^{2(d-2)+d p}}\left(I \times \mathbb{R}^{d}\right)}\right) \\
& \cdot\left(\|u\|_{L_{t}^{p+2} L_{x}^{p}}^{\frac{d p(p+2)}{4}}\left(I \times \mathbb{R}^{d}\right)\right) \\
& \leq\left\|u_{0}\right\|_{H_{x}^{s_{c}}}+C(d, p)\left(2 \eta+2 C(d, p)\left\|u_{0}\right\|_{L_{x}^{2}}\right) \\
& \cdot\left\|\left.\nabla\right|^{s_{c}} u\right\|_{L_{t}^{p+2}}^{p} \frac{2 d(p+2)}{L_{x}^{2(d-2)+d p}}\left(I \times \mathbb{R}^{d}\right) \\
& \leq\left\|u_{0}\right\|_{H_{x}^{s_{c}}}+C(d, p)\left(2 \eta+2 C(d, p)\left\|u_{0}\right\|_{L_{x}^{2}}\right)(2 \eta)^{p} .
\end{aligned}
$$

And similarly, 


$$
\begin{aligned}
& \|\Phi(u)\|_{L_{t}^{p+2}} \frac{2 d(p+2)}{L_{x}^{2(d-2)+d p}}\left(I \times \mathbb{R}^{d}\right) \\
& \leq C(d, p)\left\|u_{0}\right\|_{L_{x}^{2}}+C(d, p)\|F(u)\| \|_{L_{t}^{p+1}}^{\frac{p+2}{p+2}} \frac{2 d(p+2)}{2(d+2)+d p}\left(I \times \mathbb{R}^{d}\right) \\
& \leq C(d, p)\left\|u_{0}\right\|_{L_{x}^{2}}+C(d, p)\left\|u_{0}\right\|_{L_{x}^{2}}(2 \eta)^{p} .
\end{aligned}
$$

Arguing as above and invoking (1.4), we obtain

$$
\begin{aligned}
& \left\|\left.\nabla\right|^{s_{c}} \Phi(u)\right\|_{L_{t}^{p+2}} \frac{2 d(p+2)}{L_{x}^{2(d-2)+d p}}\left(I \times \mathbb{R}^{d}\right) \\
& \leq \eta+C(d, p)\left\|\left.\nabla\right|^{s_{c}} F(u)\right\|_{L_{t}^{p+2}} \frac{2 d(p+2)}{L_{x}^{2(d+2)+d p}}\left(I \times \mathbb{R}^{d}\right) \\
& \leq \eta+C(d, p)(2 \eta)^{1+p} .
\end{aligned}
$$

Thus, choosing $\eta_{0}=\eta_{0}(d)$ suciently small, we see that for $0<\eta \leq \eta_{0}$, the functional $\Phi$ maps the set $B_{1} \cap B_{2}$ back to itself. To see that $\Phi$ is a contraction, we repeat the above calculations to obtain

$$
\begin{aligned}
& \|\Phi(u)-\Phi(v)\|_{L_{t}^{p+2} L_{x}^{2(d-2)+d p}\left(I \times \mathbb{R}^{d}\right)} \\
& \leq C(d, p)\|F(u)-F(v)\|_{L_{t}^{p+1}}^{\frac{p+2}{L_{x}^{2}} \frac{2 d(p+2)+d p}{2(d+2)}\left(I \times \mathbb{R}^{d}\right)} \\
& \leq C(d, p)(2 \eta)^{p}\|u-v\|_{L_{t}^{p+2}} \frac{2 d(p+2)}{L_{x}^{2(d-2)+d p}}\left(I \times \mathbb{R}^{d}\right) .
\end{aligned}
$$

Therefore, choosing $\eta_{0}=\eta_{0}(d)$ even smaller (if necessary), we can ensure that $\Phi$ is a contraction on the set $B_{1} \cap B_{2}$. By the contraction mapping theorem, it follows that $\Phi$ has a fixed point in $B_{1} \cap B_{2}$. Furthermore, noting that $\Phi$ maps into $C_{t}^{0} H_{x}^{s_{c}}$ (not just $H_{x}^{s_{c}}$ ). We now turn our attention to the uniqueness. Since uniqueness is a local property, it enough to study a neighbourhood of $t=0$. By Definition of solution (and the Strichartz inequality), any solution to (1.1) belongs to $B_{1} \cap B_{2}$ on some such neighbourhood. Uniqueness thus follows from uniqueness in the contraction mapping theorem.

The claims (1.6) and (1.7) follow from another application of the Strichartz inequality.

Remark 2.1 By the Strichartz inequality, we know that

$$
\left\||\nabla|^{s_{c}} \mathrm{e}^{i t \Delta} u_{0}\right\|_{L_{t}^{p+2} L_{x}^{2(d-2)+d p}\left(\mathbb{R} \times \mathbb{R}^{d}\right)} \leq\left\|\left.\nabla\right|^{s_{c}} u_{0}\right\|_{L_{x}^{2}} .
$$

Thus, (1.4) holds with $I=\mathbb{R}$ for initial data with suciently small norm instead that, by the monotone convergence theorem, (1.4) holds provided $I$ is chosen suciently small. Note that by scaling, the length of the interval $I$ depends on the fine properties of $u_{0}$, not only on its norm.

\section{Stability of the Mass Critical}

In this section we discuss the stability theory at mass critical case. Consider the initial-value problem (1.1) with $p=\frac{4}{d}$.An important part of the local well-posedness theory is the study of how the strong solutions built in the past subsection depend upon the initial data. More accurate, we want to know if the small perturbation of the initial data gives small changes in solution. In general, we take care in developing a stability theory for nonlinear Schrödinger Equation (1.1). Even though stability is a local question, it plays an important role in all existing treatments of the global well-posedness problem for nonlinear Schrödinger equation at critical case, for more see [7]. It has also proved useful in the treatment of local and global questions for more exotic nonlinearities [8,9]. In this section, we will only discus the stability theory for the mass-critical NLS.

Lemma 3.1 Let $I$ be a compact interval and let $\tilde{u}$ be an approximate solution to (1.1) meaning that

$$
\left(i \partial_{t}+\Delta\right) \tilde{u}=F(\tilde{u})+e,
$$

for some function $e$. Suppose that

$$
\tilde{u}_{L_{t}^{\infty} L_{x}^{2}\left(I \times \mathbb{R}^{d}\right)} \leq M
$$

for some positive constant $M$. Let $t_{0} \in I$ and let $u\left(t_{0}\right)$ be such that

$$
\left\|u\left(t_{0}\right)-\tilde{u}\left(t_{0}\right)\right\|_{L_{x}^{2}} \leq M^{\prime}
$$

for some $M^{\prime}>0$. Suppose also the smallness conditions

$$
\begin{aligned}
& \|\tilde{u}\|_{L_{t, x}{ }^{\frac{2(d+2)}{d}}\left(I \times \mathbb{R}^{d}\right)} \leq \varepsilon_{0} \\
& \left.\left\|\mathrm{e}^{i\left(t-t_{0}\right) \Delta}\left(u\left(t_{0}\right)-\tilde{u}\left(t_{0}\right)\right)\right\|_{L_{t, x}{ }^{d}} \sum_{(d \times 2)} \leq \mathbb{R}^{d}\right) \\
& \|e\|_{N^{0}(I)} \leq \varepsilon
\end{aligned}
$$

for some $0<\varepsilon \leq \varepsilon_{0}$ where $\varepsilon_{0}=\varepsilon_{0}\left(M, M^{\prime}\right)>0$ is a small constant. Then, there exists a solution $u$ to (1.1) on $\left(I \times \mathbb{R}^{d}\right)$ with initial data $u\left(t_{0}\right)$ at time $t=t_{0}$ satisfying

$$
\begin{aligned}
& \|u-\tilde{u}\|_{L_{t, x^{d}}}^{\frac{2(d+2)}{\left(I \times \mathbb{R}^{d}\right)}} \lesssim \varepsilon \\
& \|u-\tilde{u}\|_{S^{0}(I)} \lesssim M^{\prime} \\
& \|u\|_{S^{0}(I)} \lesssim M+M^{\prime} \\
& \|F(u)-F(\tilde{u})\|_{N^{0}(I)} \lesssim \varepsilon
\end{aligned}
$$

Proof: By symmetry, we may assume $t_{0}=\inf I$. Let $w:=u-\tilde{u}$. Then $w$ satisfies the initial value problem

$$
\left\{\begin{array}{l}
i w_{t}+\Delta w=F(\tilde{u}+w)-F(\tilde{u})-e \\
w\left(t_{0}\right)=u\left(t_{0}\right)-\tilde{u}\left(t_{0}\right)
\end{array}\right.
$$

For $t \in I$ we define 


$$
A(t):=\|F(\tilde{u}+w)-F(\tilde{u})\|_{N^{0}\left(\left[t_{0}, t\right]\right)} .
$$

By (3.3),

$$
\begin{aligned}
& A(t) \lesssim\|F(\tilde{u}+w)-F(\tilde{u})\|_{L_{t, x} \frac{2(d+2)}{d+4}\left(\left[t_{0}, t\right] \times \mathbb{R}^{d}\right)} \\
& \lesssim\|w\|_{\frac{2}{2(d+2)}}^{\substack{1+\frac{4}{d} \\
L_{t, x^{d}}}}\left(\left[t_{0}, t\right] \times \mathbb{R}^{d}\right)
\end{aligned}
$$

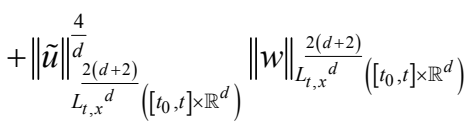

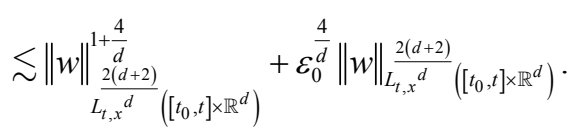

Furthermore, by Strichartz, (3.4), and (3.5), we get

$$
\begin{aligned}
& \|w\|_{L_{t, x} x^{d}}^{\frac{2(d+2)}{\left(\left[t_{0}, t\right] \times \mathbb{R}^{d}\right)}} \lesssim\left\|\mathrm{e}^{i\left(t-t_{0}\right) \Delta} w\left(t_{0}\right)\right\|_{L_{t, x^{d}}}^{\frac{2(d+2)}{\left(t^{d}\right.}}\left(\left[t_{0}, t\right] \times \mathbb{R}^{d}\right) \\
& +A(t)+\|e\|_{N^{0}\left(\left[t_{0}, t\right]\right)} \\
& \lesssim A(t)+\varepsilon \text {. }
\end{aligned}
$$

Combining (3.10) and (3.11), we obtain

$$
A(t) \lesssim(A(t)+\varepsilon)^{1+\frac{4}{d}}+\varepsilon_{0}^{\frac{4}{d}}(A(t)+\varepsilon)+\varepsilon .
$$

A standard continuity argument then shows that if $\varepsilon_{0}$ is taken sufficiently small,

$$
A(t) \lesssim \varepsilon \text { for any } t \in I,
$$

which implies (3.9). Using (3.9) and (3.11), we obtained (3.6). Furthermore, by Strichartz, (3.2), (3.5), and (3.9),

$$
\begin{aligned}
\|w\|_{S^{0}(I)} & \lesssim\left\|w\left(t_{0}\right)\right\|_{L_{x}^{2}}+\|F(\tilde{u}+w)-F(\tilde{u})\|_{N^{0}(I)}\|e\|_{N^{0}(I)} \\
& \lesssim M^{\prime}+\varepsilon,
\end{aligned}
$$

which establishes (3.7) for $\varepsilon_{0}=\varepsilon_{0}\left(M^{\prime}\right)$ sufficiently small.

To prove (3.8), we use Strichartz, (3.1), (3.2), (3.9), and (3.3):

$$
\begin{aligned}
\|u\|_{S^{0}(I)} \lesssim & \left\|u\left(t_{0}\right)\right\|_{L_{x}^{2}}+\|F(u)\|_{N^{0}(I)} \\
\lesssim & \left\|\tilde{u}\left(t_{0}\right)\right\|_{L_{x}^{2}}+\left\|u\left(t_{0}\right)-\tilde{u}\left(t_{0}\right)\right\|_{L_{x}^{2}} \\
& +\|F(u)-F(\tilde{u})\|_{N^{0}(I)}+\|F(\tilde{u})\|_{N^{0}(I)} \\
\lesssim & M+M^{\prime}+\varepsilon+\|u\|_{L_{t, x^{d}}+\frac{4}{d}}^{\frac{2(d+2)}{\left(I \times \mathbb{R}^{d}\right)}} \\
\lesssim & M+M^{\prime}+\varepsilon+\varepsilon_{0}^{1+\frac{4}{d}} .
\end{aligned}
$$

Choosing $\varepsilon_{0}=\varepsilon_{0}\left(M, M^{\prime}\right)$ sufficiently small, this finishes the proof.

Based on the previous result, we are now able to prove stability for the mass-critical NLS.
Theorem 3.2 Let $I$ be a compact interval and let $\tilde{u}$ be an approximate solution to (1.1) in the sense that

$$
\left(i \partial_{t}+\Delta\right) \tilde{u}=F(\tilde{u})+e
$$

for some function $e$. Assume that condition (3.1) in Lemma 3.1 holds and

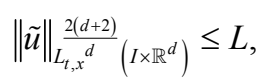

for some positive constant $M$ and $N$. Let $t_{0} \in I$ and let $u\left(t_{0}\right)$ obey (3.2) for some $M^{\prime}>0$. Furthermore, suppose the smallness conditions (3.4), (3.5) in Lemma 3.1. For some $0<\varepsilon \leq \varepsilon_{1}$ where

$\varepsilon_{1}=\varepsilon_{1}\left(M, M^{\prime}, L\right)>0$ is a small constant. Then, there exists a solution $u$ to $(1.1)$ on $\left(I \times \mathbb{R}^{d}\right)$ with initial data $u\left(t_{0}\right)$ at time $t=t_{0}$ satisfying

$$
\begin{aligned}
& \|u-\tilde{u}\|_{L_{t, x^{\prime}} \|^{2(d+2)}}\left(I \times \mathbb{R}^{d}\right) \\
& \|u-\tilde{u}\|_{S^{0}(I)} \lesssim C C\left(M, M^{\prime}, L\right) M^{\prime} \\
& \|u\|_{S^{0}(I)} \lesssim C\left(M, M^{\prime}, L\right)
\end{aligned}
$$

Proof: Subdivide $I$ into $J \sim\left(1+\frac{L}{\varepsilon_{0}}\right)^{\frac{2(d+2)}{d}}$ subintervals $I_{j}=\left[t_{j}, t_{j+1}\right], 0 \leq j<J$, such that

$$
\|\tilde{u}\|_{L_{t, x^{d}}{ }^{d}}^{2(d+2)}\left(I_{j} \times \mathbb{R}^{d}\right) \leq \varepsilon_{0}
$$

where $\varepsilon_{0}=\varepsilon_{0}\left(M, 2 M^{\prime}\right)>0$ is as in Lemma 3.1. We replaced $M^{\prime}$ by $2 M^{\prime}$ as the mass of the difference $u-\tilde{u}$ might grow slightly in time. By choosing $\varepsilon_{1}$ sufficiently small depending on $J, M$ and $M^{\prime}$, we can apply Lemma 3.1 to obtain for each $j$ and all $0<\varepsilon \leq \varepsilon_{1}$

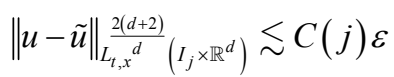

$$
\begin{aligned}
& \|u-\tilde{u}\|_{S^{0}\left(I_{j}\right)} \lesssim C(j) M^{\prime} \\
& \|u\|_{S^{0}\left(I_{j}\right)} \lesssim C(j)\left(M+M^{\prime}\right) \\
& \|F(u)-F(\tilde{u})\|_{N^{0}\left(I_{j}\right)} \lesssim C(j) \varepsilon .
\end{aligned}
$$

Provided, we can prove that their counterparts of (3.2) and (3.4) hold with replace $t_{0}$ by $t_{j}$. To verify this, we use an inductive argument. By Strichartz, (3.2), (3.5), and the inductive hypothesis,

$$
\begin{aligned}
& \left\|u\left(t_{j}\right)-\tilde{u}\left(t_{j}\right)\right\|_{L_{x}^{2}} \lesssim\left\|u\left(t_{0}\right)-\tilde{u}\left(t_{0}\right)\right\|_{L_{x}^{2}} \\
& +\|F(u)-F(\tilde{u})\|_{N^{0}\left(\left[t_{0}, t_{j}\right]\right)}+\|e\|_{N^{0}\left(\left[t_{0}, t_{j}\right]\right)} \\
& \lesssim M^{\prime}+\sum_{k=0}^{j-1} C(k) \varepsilon+\varepsilon .
\end{aligned}
$$


Similarly, by Strichartz, (3.4), (3.4), and the inductive hypothesis,

$$
\begin{aligned}
& \left\|\mathrm{e}^{i\left(t-t_{j}\right) \Delta}\left(u\left(t_{j}\right)-\tilde{u}\left(t_{j}\right)\right)\right\|_{L_{t, x^{d}}}\left(\frac{2(d+2)}{\left.I_{j} \times \mathbb{R}^{d}\right)}\right. \\
& \left.\lesssim\left\|\mathrm{e}^{i\left(t-t_{0}\right) \Delta}\left(u\left(t_{0}\right)-\tilde{u}\left(t_{0}\right)\right)\right\|_{L_{t, x^{d}}}\right|_{(I \times 2)}\left(I \times \mathbb{R}^{d}\right) \\
& +\|e\|_{N^{0}\left(\left[t_{0}, t_{j}\right]\right)}+\|F(u)-F(\tilde{u})\|_{N^{0}\left(\left[\left[_{0}, t_{j}\right]\right)\right.} \\
& \lesssim \varepsilon+\sum_{k=0}^{j-1} C(k) \varepsilon .
\end{aligned}
$$

Choosing $\varepsilon_{1}$ sufficiently small depending on $J, M$, and $M^{\prime}$, we can ensure that hypotheses of Lemma 3.1 continue to hold as $j$ varies.

Lemma 3.3 (Stability) Fix $u$ and $d$. For every $A>0$ and $\varepsilon>0$ there exists $\delta>0$ with the property: if $u: I \times \mathbb{R}^{d} \rightarrow C$ is such that $S(u) \leq A$ and that $u$ approximately solves (1.1) in the sense that

$$
\left\|i u_{t}+\Delta u-F(u)\right\|_{L_{t, x}^{2(d+2) /(d+4)}\left(I \times \mathbb{R}^{d}\right)} \leq \delta .
$$

And $t_{0} \in I, \quad v_{0} \in L_{x}^{2}\left(\mathbb{R}^{d}\right)$ are such that

$$
S_{I}\left(\mathrm{e}^{i\left(t-t_{0}\right) \Delta}\left(u\left(t_{0}\right)-v_{0}\right)\right) \leq \delta^{2(d+2) / d},
$$

Then there exists a solution $v: I \times \mathbb{R}^{d} \rightarrow C$ to (1.1) with $v\left(t_{0}\right)=v_{0}$ such that

$$
S(u-v) \leq \varepsilon .
$$

Note that, the masses of $u$ and $v_{0}$ do not appear immediately in this lemma, although it is necessary that these masses are finite. Similar stability results for the energy-critical NLS (in $\dot{H}^{1} \mathbb{R}^{d}$ ) instead of $L_{x}^{2}\left(\mathbb{R}^{d}\right)$, of course) have appeared in [10-14]. The mass-critical case it is actually slightly simpler as one does not need to deal with the existence of a derivative in the regularity class. For more see [15].

Proof: (Sketch) First let prove the claim when $A$ is suciently small depending on $d$. Let $v: I^{\prime} \times \mathbb{R}^{d} \rightarrow C$ be the maximal-lifespan solution with initial data $v\left(t_{0}\right)=v_{0}$. Writing $v=u+w$ on the interval $I^{\prime \prime}:=I \cap I^{\prime}$, we see that

$$
i w_{t}+\Delta w=(F(u+w)-F(u))-\left(i u_{t}+\Delta u-F(u)\right)
$$

and

$$
S_{I^{\prime \prime}}\left(\mathrm{e}^{i\left(t-t_{0}\right) \Delta} w\left(t_{0}\right)\right) \leq \delta^{2(d+2) / d} .
$$

Thus, if we set

$$
X:=\|w\|_{L_{t, x}^{2(d+2) / d}\left(I^{\prime \prime} \times \mathbb{R}^{d}\right)}
$$

by the triangle inequality, (2.3), and (3.16), we have

$$
X \leq \delta+C_{d}^{\prime \prime}\left[\|F(u+w)-F(u)\|_{L_{t, x}^{2(d+2)((d+4)}\left(I^{\prime \prime} \times \mathbb{R}^{d}\right)}+\delta\right],
$$

hence, by (2.4) and the hypothesis $S(u) \leq A$,

$$
X \leq \tilde{C}_{d}\left[A^{2 /(d+2)} X+X^{1+4 / d}+\delta\right]
$$

where $\tilde{C}_{d}$ depends only on $d$. If $A$ is suciently small depending on $d$, and $\delta$ is suciently small depending on $\varepsilon$ and $d$, then standard continuity arguments give $X \leq \varepsilon$ as desired. To deal with the case when $A$ is large, simply iterate the case when $A$ is small (shrinking $\delta, \varepsilon$ repeatedly) after a subdivision of the time interval $I$.

\section{Stability of the Mass-Supercritical and Energy-Subcritical}

In this section we discuss the Stability theory of the mass-supercritical and energy-subcritical to the nonlinear Schrödinger equation. Consider the initial-value problem (1.1) with $p=2$ and $d=3$ we chose $\mu=-1$.

In this case the initial-value problem $u=u(x, 0)=u_{0}$ is locally well-posed in $H^{1}$. Now we rewrite (1.1) as

$$
\left\{\begin{array}{l}
i u_{t}+\Delta u+|u|^{2} u=0 \\
u(x, 0)=u_{0}(x) \in H^{1}\left(R^{3}\right)
\end{array}\right\}
$$

We discuss the stability by the following proposition. Before beginning we need define the Kato inhomogeneous Strichartz estimate. See [16]

$$
\left\|\int_{0}^{t} \mathrm{e}^{i\left(t-t^{\prime}\right) \Delta} f\left(., t^{\prime}\right) \mathrm{d} t\right\|_{S\left(\dot{H}^{1 / 2}\right)} \leq c\|f\|_{S^{\prime}\left(\dot{H}^{-1 / 2}\right)}
$$

Proposition 4.1 For each $N \square 1$ there exists $\varepsilon_{0}=\varepsilon_{0}(N) \square 1$, and $c=c(N) \square 1$, such that the following holds.

Let $u=u(x, t) \in H_{x}^{1}$ for all $t$ and solve

$$
i \partial_{t} u+\Delta u+|u|^{2} u=0 \text {. }
$$

Let $\tilde{u}=\tilde{u}(x, t) \in H_{x}^{1}$ for all $t$ and define

If

$$
e=i \partial_{t} \tilde{u}+\Delta \tilde{u}+|\tilde{u}|^{2} \tilde{u} .
$$

$$
\|\tilde{u}\|_{S\left(\dot{H}^{1 / 2}\right)} \leq N,\|e\|_{S^{\prime}\left(\dot{H}^{-1 / 2}\right)} \leq \varepsilon_{0} .
$$

And

$$
\left\|\mathrm{e}^{i\left(t-t_{0}\right) \Delta}\left(u\left(t_{0}\right)-\tilde{u}\left(t_{0}\right)\right)\right\|_{S\left(\dot{H}^{1 / 2}\right)} \leq \varepsilon_{0} .
$$

Then

$$
\|u\|_{S\left(\dot{H}^{1 / 2}\right)} \leq c=c(N)<\infty .
$$

Proof: Let $w$ be defined by $u=\tilde{u}+w$. then $w$ solves the equation

$$
\begin{aligned}
& i \partial_{t} w+\Delta w+\left(\tilde{u}^{2} \bar{w}+2|\tilde{u}|^{2} w\right) \\
& +\left(2 \tilde{u}|w|^{2}+\overline{\tilde{u}} w^{2}\right)+|w|^{2} w-e=0
\end{aligned}
$$


since $\|\tilde{u}\|_{S\left(\dot{H}^{1 / 2}\right)} \leq N$.Can be divided $\left[t_{0}, t_{\infty}\right)$ into $A=A(N)$ in intervals $I_{j}=\left[t_{j}, t_{j+1}\right]$ Such that for all $j$, the quantity $\|\tilde{u}\|_{S\left(\dot{H}^{1 / 2}, I_{j}\right)} \leq \delta$, is Appropriate small ( $\delta$ to be selected below).

Integration (4.3) with initial time $t_{j}$ is

$$
w(t)=\mathrm{e}^{i\left(t-t_{j}\right) \Delta} w\left(t_{j}\right)+i \int_{t_{j}}^{t} \mathrm{e}^{i(t-s) \Delta} \mathcal{W}(., s) \mathrm{d} s
$$

where

$$
\mathcal{W}=\left(\tilde{u}^{2} \bar{w}+2|\tilde{u}|^{2} w\right)+\left(2 \tilde{u}|w|^{2}+\overline{\tilde{u}} w^{2}\right)+|w|^{2} w-e .
$$

Applying the Kato Strichartz estimate (4.1) on $I_{j}$, to obtain

$$
\begin{aligned}
\|w\|_{S\left(\dot{H}^{1 / 2}, I_{j}\right)} \leq & \left\|\mathrm{e}^{i\left(t-t_{j}\right) \Delta} w\left(t_{j}\right)\right\|_{S\left(\dot{H}^{1 / 2}, I_{j}\right)} \\
& +c\left\|\tilde{u}^{2} w\right\|_{L_{L_{j}}^{3}}^{\frac{10}{4}}+c\left\|\tilde{u} w^{2}\right\|_{L_{L_{j}}^{3}}^{\frac{10}{4}} \frac{5}{L_{x}^{4}} \\
& +c\left\|w^{3}\right\|_{L_{I_{j}}^{3}}^{\frac{10}{3}} \frac{5}{L_{x}^{4}}+\|e\|_{S^{\prime}\left(\dot{H}^{-1 / 2}, I_{j}\right)}
\end{aligned}
$$

Note that

$$
\begin{aligned}
\left\|\tilde{u}^{2} w\right\|_{L_{I_{j}}^{3}}^{\frac{10}{L_{x}^{4}}} & \leq\|\tilde{u}\|_{L_{I_{j}}^{20} \frac{10}{L_{x}^{3}}}^{2}\|w\|_{L_{I_{j}}^{5} L_{x}^{5}} \\
& \leq\|\tilde{u}\|_{S\left(\dot{H}^{1 / 2}, I_{j}\right)}^{2}\|w\|_{S\left(\dot{H}^{1 / 2}, I_{j}\right)} . \\
& \leq \delta^{2}\|w\|_{S\left(\dot{H}^{1 / 2}, I_{j}\right)}
\end{aligned}
$$

Similarly,

$$
\left\|\tilde{u} w^{2}\right\|_{L_{I_{j}}^{3}}^{10} \frac{5}{4} \leq \delta\|w\|_{S\left(\dot{H}_{x}^{1 / 2}, I_{j}\right)}^{2}
$$

and

$$
\left\|w^{3}\right\|_{L_{I_{j}}^{3}}^{\frac{10}{3}} \frac{5}{4} \leq\|w\|_{S\left(\dot{H}^{1 / 2}, I_{j}\right)}^{3} .
$$

Substituting the above estimates in (4.5), to get,

$$
\begin{aligned}
\|w\|_{S\left(\dot{H}^{1 / 2}, I_{j}\right)} \leq & \left\|\mathrm{e}^{i\left(t-t_{j}\right) \Delta} w\left(t_{j}\right)\right\|_{S\left(\dot{H}^{1 / 2}, I_{j}\right)}+c \delta^{2}\|w\|_{S\left(\dot{H}^{1 / 2}, I_{j}\right)} \\
& +c \delta\|w\|_{S\left(\dot{H}^{1 / 2}, I_{j}\right)}^{2}+c\|w\|_{S\left(\dot{H}^{1 / 2}, I_{j}\right)}^{3}+c \varepsilon_{0} .
\end{aligned}
$$

As long as

$$
\delta \leq \min \left(1, \frac{1}{6 c}\right)
$$

and

$$
\left(\left\|\mathrm{e}^{i\left(t-t_{j}\right) \Delta} w\left(t_{j}\right)\right\|_{S\left(\dot{H}^{1 / 2}, I_{j}\right)}+c \varepsilon_{0}\right) \leq \min \left(1, \frac{1}{2 \sqrt{6 c}}\right) .
$$

We obtain

$$
\|w\|_{S\left(\dot{H}^{1 / 2}, I_{j}\right)} \leq 2\left\|\mathrm{e}^{i\left(t-t_{j}\right) \Delta} w\left(t_{j}\right)\right\|_{S\left(\dot{H}^{1 / 2}, I_{j}\right)}+2 c \varepsilon_{0} .
$$

Taken now $t=t_{j+1}$ in (4.4) and apply $\mathrm{e}^{i\left(t-t_{j+1}\right) \Delta}$ to both sides to obtain

$$
\begin{aligned}
& \mathrm{e}^{i\left(t-t_{j+1}\right) \Delta} w\left(t_{j+1}\right) \\
& =\mathrm{e}^{i\left(t-t_{j}\right) \Delta} w\left(t_{j}\right)+i \int_{t_{j}}^{t_{j+1}} \mathrm{e}^{i(t-s) \Delta} \mathcal{W}(., s) \mathrm{d} s .
\end{aligned}
$$

Since the Duhamel integral is restricted to $I_{j}=\left[t_{j}, t_{j+1}\right]$, by again applying the Kato estimate, similarly to (4.6) we obtain,

$$
\begin{aligned}
& \left\|\mathrm{e}^{i\left(t-t_{j+1}\right) \Delta} w\left(t_{j+1}\right)\right\|_{S\left(\dot{H}^{1 / 2}, I_{j}\right)} \\
& \leq\left\|\mathrm{e}^{i\left(t-t_{j}\right) \Delta} w\left(t_{j}\right)\right\|_{S\left(\dot{H}^{1 / 2}, I_{j}\right)}+c \delta^{2}\|w\|_{S\left(\dot{H}^{1 / 2}, I_{j}\right)} \\
& \quad+c \delta\|w\|_{S\left(\dot{H}^{1 / 2}, I_{j}\right)}^{2}+c\|w\|_{S\left(\dot{H}^{1 / 2}, I_{j}\right)}^{3}+c \varepsilon_{0} .
\end{aligned}
$$

By (4.8) and (4.9), we bound the Former of expression to obtain

$$
\begin{aligned}
& \left\|\mathrm{e}^{i\left(t-t_{j+1}\right) \Delta} w\left(t_{j+1}\right)\right\|_{S\left(\dot{H}^{1 / 2}, I_{j}\right)} \\
& \leq 2\left\|\mathrm{e}^{i\left(t-t_{j}\right) \Delta} w\left(t_{j}\right)\right\|_{S\left(\dot{H}^{1 / 2}, I_{j}\right)}+2 c \varepsilon_{0} .
\end{aligned}
$$

Start iterates with $j=0$, we obtain

$$
\begin{aligned}
& \left\|\mathrm{e}^{i\left(t-t_{j}\right) \Delta} w\left(t_{j}\right)\right\|_{S\left(\dot{H}^{1 / 2}, I_{j}\right)} \\
& \leq 2^{j}\left\|\mathrm{e}^{i\left(t-t_{0}\right) \Delta} w\left(t_{0}\right)\right\|_{S\left(\dot{H}^{1 / 2}, I_{j}\right)}+\left(2^{j}-1\right) 2 c \varepsilon_{0} \\
& \leq 2^{j+2} c \varepsilon_{0} .
\end{aligned}
$$

To absorb the second part of (4.7) for all intervals $I_{j}$, $0 \leq j \leq A-1$, we require

$$
2^{A+2} c \varepsilon_{0} \leq \min \left(1, \frac{1}{2 \sqrt{6 c}}\right) .
$$

We review that the dependence of parameters $\delta$ is an absolute constant chosen to meet the first part of (4.7). The inequality (4.10) determines how the small $\varepsilon_{0}$ needs to be taken in terms of $A$ (and thus, in terms of $N$ ). We were given $N$ which then determined $A$.

\section{REFERENCES}

[1] S. Keraani, "On the Blow-Up Phenomenon of the Critical Nonlinear Schrödinger Equation," Journal of Functional Analysis, Vol. 235, No. 1, 2006, pp. 171-192. doi:10.1016/j.jfa.2005.10.005

[2] R. Killip, M. Visan and X. Zhang, "Energy-Critical NLS 
with Quadratic Potentials," Communications in Partial Differential Equations, Vol. 34, No. 12, 2009, pp. 15311565.

[3] T. Tao, M. Visan and X. Zhang, "Global Well-Posedness and Scattering for the Mass-Critical Nonlinear Schrödinger Equation for Radial Data in High Dimensions," Duke Mathematical Journal, Vol. 140, No. 1, 2007, pp. 165-202.

[4] T. Cazenave, "Semilinear Schrödinger Equations," Courant Lecture Notes in Mathematics, New York University, 2003.

[5] T. Cazenave and F. B. Weissler, "Critical Nonlinear Schrödinger Equation," Nonlinear Analysis: Theory, Methods \& Applications, Vol. 14, No. 10, 1990, pp. 807-836. doi:10.1016/0362-546X(90)90023-A

[6] Y. Tsutsumi, " $\mathrm{L}^{2}$-Solutions for Nonlinear Schrödinger Equations and Nonlinear Groups," Funkcial Ekvac, Vol. 30, No. 1, 1987, pp. 115-125.

[7] R. Killip and M. Visan, "Nonlinear Schrödinger Equations at Critical Regularity," Clay Mathematics Proceedings, Vol. 10, 2009.

[8] T. Tao, M. Visan and X. Zhang, "The Nonlinear Schrödinger Equation with Combined Power-Type Nonlinearities," Communications in Partial Differential Equations, Vol. 32, No. 7-9, 2007, pp. 1281-1343.

[9] X. Zhang, "On the Cauchy Problem of 3-D EnergyCritical Schrödinger Equations with Sub-Critical Perturbations," Journal of Differential Equations, Vol. 230, No.
2, 2006, pp. 422-445.

[10] J. Colliander, M. Keel, G. Stafflani, H. Takaoka and T. Tao, "Global Well-Posedness and Scattering in the Energy Space for the Critical Nonlinear Schrödinger Equation in $\mathrm{R}^{3}$," Analysis of PDEs.

[11] C. Kenig and F. Merle, "Global Well-Posedness, Scattering, and Blowup for the Energy-Critical, Focusing, Non-Linear Schrödinger Equation in the Radial Case," preprint.

[12] E. Ryckman and M. Visan, "Global Well-Posedness and Scattering for the Defocusing Energy-Critical Nonlinear Schrödinger Equation in $\mathrm{R}^{1+4}$," American Journal of Mathematics.

[13] T. Tao and M. Visan, "Stability of Energy-Critical Nonlinear Schrödinger Equations in High Dimensions," Electronic Journal of Differential Equations, Vol. 2005, No. 118, 2005, pp. 1-28.

[14] M. Visan, "The Defocusing Energy-Critical Nonlinear Schrödinger Equation in Higher Dimensions," Duke Mathematical Journal.

[15] T. Tao, M. Visan and X. Zhang, "Minimal-Mass Blowup Solutions of the Mass-Critical NLS," Forum Mathematicum, Vol. 20, No. 5, 2008, pp. 881-919. doi:10.1515/FORUM.2008.042

[16] D. Foschi, "Inhomogeneous Strichartz Estimates," Advances in Difference Equations, Vol. 2, No. 1, 2005, pp. 124. 